\title{
Forced expression of heat-shock protein 70 increases the secretion of Hsp70 and provides protection against tumour
} growth

\author{
M-H Wang', ME Grossmann' and CYF Young*,I \\ 'Department of Urology and Biochemistry/Molecular Biology, Mayo Graduate School, Mayo Clinic/Foundation, Rochester, MN 55905, USA
}

\begin{abstract}
Although heat-shock protein 70 (Hsp70) has been considered an intracellular protein, we report that Hsp70 is secreted under normal cell culture conditions by human prostate cell lines, LAPC-4, PC-3, CWR-22, RWPE-I and -2, LNCaP, and TRAMP (transgenic adenocarcinoma mouse prostate)-C2. We found that the secretion can be enhanced by transfection with cDNA encoding for Hsp70. To verify that the Hsp70 detected in the supernatant was not secondary to cell leakage, C2 cells were cotransfected with cytoplasmic Renilla luciferase as a reporter. High levels of activities were noted in the cell extracts, while no enzyme activities were detected in the supernatants. To verify that forced oversecretion of Hsp70 could protect against tumour growth, mice were injected with $\mathrm{C} 2$ cells transfected with an Hsp70 DNA construct and challenged with live tumour cells. Mice injected with cells transfected with the Hsp70 DNA construct demonstrated a significantly decreased rate of tumour growth compared to those injected with empty vector. In addition, a difference in survival rate as defined by a surrogate end point was noted between the two groups. In a second experiment, we developed a cell line that stably overexpressed Hsp70. Mice injected with these cells also demonstrated a significant decrease in tumour growth and significantly increased survival.

British Journal of Cancer (2004) 90,926-93I. doi:I0.1038/sj.bjc.660I583 www.bjcancer.com

(c) 2004 Cancer Research UK
\end{abstract}

Keywords: heat-shock protein 70; secretion; prostate cancer; transgenic adenocarcinoma mouse prostate; gene therapy

Prostate cancer is the second leading cause of cancer death in men in the United States, exceeded only by lung cancer. The American Cancer Society estimates that 28900 men in the United States will die of prostate cancer in the year 2003. Currently, for patients with early stage, organ confined disease, there are well-defined treatment options, including radical prostatectomy, radiation therapy, or watchful waiting. However, no definitive treatments are available for advanced or recurrent disease. It is known that tumour regression can be achieved with androgen blockade; however, disease usually recurs within 1-2 years, leading to significant morbidity and mortality (Mahler and Denis, 1992).

The idea of gene transfer to enable the use of host immune system against tumours has generated new treatment options for patients with prostate cancer. This therapy is based on the assumption that it is possible to break tolerance to tumour antigens by increased expression of immunomodulants and chemokines (Houghton, 1994). Specifically in prostate cancer studies, various gene transfer strategies using human or murine granulocyte/macrophage colony-stimulating factor (Sanda et al, 1994), interleukin-2 (Fearon et al, 1990), and interferon gamma (Vieweg et al, 1994) have been shown to elicit antitumour responses.

The antitumour property of heat-shock proteins (hsp's) was recognised in the 1980s when purified hsp's from tumour cells were shown to elicit immunity (Srivastava et al, 1998). Subsequent

*Correspondence: Dr CYF Young; E-Mail: youngc@mayo.edu Received 13 August 2003; revised 30 October 2003; accepted 20 November 2003 studies have contributed to the understanding of the mechanism by which purified hsp's interact with the immune system. In their role as housekeeping proteins and chaperones, hsp's can bind to multiple intracellular peptides including tumour peptides (Gething and Sambrook, 1992; Parsell and Lindquist, 1993). These hsp-peptide complexes have the unique ability to promote crosspriming of cytotoxic T lymphocytes (CTLs), one of the most effective ways to stimulate antitumour immunity (Cavallo et al, 1993; Suto and Srivastava, 1995; Cayeux et al, 1997). Once released from tumour cells, these hsp complexes bind to CD91 receptors on host antigen-presenting cells (APCs) (Binder et al, 2000). The binding of hsp-peptide complex with CD91 leads to the internalisation of the complex and presentation of tumour peptides with MHC class I with the activation of CD8 + cells. Studies also suggest that a small proportion of the hsp-peptide complex is loaded onto MHC class II, leading to the stimulation of CD4 + cells (Matsutake and Srivastava, 1999). Other receptors, such as CD36 and CD40, were identified recently on APCs that can also interact with hsp's (Panjwani et al, 2000; Wang, 2001). These receptors, once activated, cause the secretion of nonspecific inflammatory cytokines such as tumour necrosis factors and interleukins (Ishii et al, 1999).

Recent studies indicated that hsp's interact with natural killer (NK) cells. Studies have demonstrated a correlation between tumour cell hsp's expression and increased NK cell-mediated cell lysis (Ponomarev et al, 2000). This observation is further supported by the identification of the extracellular C-terminal epitopes on Hsp70, 504-617, which are important for NK cells' killing activities (Botzler et al, 1998). 
Previously in our laboratory, we found that purified Hsp70 from transgenic adenocarcinoma mouse prostate (TRAMP)-C2 cells (Foster et al, 1997) can induce an antitumour response (Vanaja et al, 2000). TRAMP-C2 is a murine prostate cancer cell line derived from TRAMP mice (transgenic adenocarcinoma of mouse prostate) that spontaneously develop prostate cancer (Greenberg et al, 1995). In the process of extending our previous studies on hsp's, we noted the presence of Hsp70 in the routine cell culture media of prostate cancer cells. This led us to investigate Hsp70 secretion and its significance in antitumour therapy. The advantage of using hsp's gene transfer is that it bypasses the need to purify large quantities of hsp's, in addition to allowing for systemic delivery.

\section{MATERIALS AND METHODS}

\section{Cell lines}

TRAMP C2 cells were cultured in Dulbecco's modified Eagle's medium (DMEM, GIBCO, CA, USA) supplemented with $5 \%$ fetal calf serum (FCS), and $1 \%$ penicillin/streptomycin. Cells were maintained in $\mathrm{T} 162 \mathrm{~cm}$ flasks at $37^{\circ} \mathrm{C}, 5 \% \mathrm{CO}_{2}$, and passaged weekly. Cells used for animal injections were collected by trypsinisation and washed with DMEM three times prior to injections. Specified number of viable cells ( $100 \mu$ l of DMEM per mouse) was determined by trypan blue exclusion, and used for injection.

LNCaP, PC-3, CWR-22, and LAPC-4 are human prostate adenocarcinoma cell lines. Each cell line was cultured in RPMI 1640 media (GIBCO, CA, USA) containing 5\% FCS and $1 \%$ penicillin/streptomycin. Cells were maintained at $37^{\circ} \mathrm{C}, 5 \% \mathrm{CO}_{2}$. RWPE-1 and RWPE- 2 cells derived from normal human prostate cells immortalised with human papilloma virus 18 were maintained in keratinocyte media (GIBCO, CA, USA), $37^{\circ} \mathrm{C}$, and $5 \%$ $\mathrm{CO}_{2}$ (Bello et al, 1997).

\section{Hsp70-expressing cells}

The full-length cDNA coding for inducible mouse Hsp70 was inserted into a mammalian expression vector pcDNA3.1 $(+)$ (Invitrogen, CA, USA), and transiently transfected into TRAMP-C2 cells as per the protocol (Superfect, Qiagen, CA, USA). Empty vector was transfected as a control. Supernatants and cell extracts were collected at 24, 48, and $72 \mathrm{~h}$. Whole-cell extracts were prepared as per Santa Cruz Biotechnology research applications. Spent media were spun down at 1000 r.p.m. for 5 min; supernatant was collected and concentrated with Vivaspin column concentrator, 10000 MWCO (Vivascience, CA, USA). Protein levels were quantified with DC protein assay or Bradford (Bio-Rad, CA, USA). Success of the transfections was verified by Western analysis for Hsp70. To generate stable clones, cells were transfected with pcDNA3.1 + Hsp70 or empty vector as above and selected with gentamicin. Positive clones were selected and verified by Western analysis for Hsp70.

\section{Secretion study}

TRAMP-C2 cells were cotransfected with pcDNA3.1 + Hsp70. and Renilla luciferase vector (Promega, WI, USA). Supernatants and cell extracts were collected at 24, 48, and $72 \mathrm{~h}$. Proteins were collected and analysed by Western analysis for Hsp70 as described below and luciferase activity was measured as per the manufacturer's instructions (Promega, WI, USA). Western blots of Hsp70 protein were quantified by densitometry and luciferase activity was measured by luminescence. All experiments were performed in triplicate.

LNCaP cells were cultured in RPMI 1640 media (GIBCO, CA, USA) containing 5\% FCS, $1 \%$ penicillin/streptomycin, and $1 \mathrm{~nm}$ mibolerone, a synthetic androgen. Various concentrations of brefeldin A (BFA, Sigma, MO, USA) dissolved in RPMI 1640 were added to each plate. At $16 \mathrm{~h}$ after the BFA treatment, both supernatants and cells were collected and prepared as above for Western analysis.

\section{Western blot analysis}

Whole-cell protein extracts and supernatants were prepared and quantified using DC assay or Bradford assay (BioRad, CA, USA). Equivalent protein samples were loaded into a precast $4-12 \%$ NuPage gel (SDS-PAGE), followed by electrophoresis and subsequent transfer onto a nitrocellulose membrane. Ponceau S staining was performed for total protein staining. The membrane was blocked overnight at $4{ }^{\circ} \mathrm{C}$ with $5 \%$ milk in PBST (phosphate buffer solution with $1 \%$ Tween 20 ) and washed five times, 5 min each with PBST. This was followed by incubating the membrane at room temperature with either inducible Hsp70 primary antibody (StressGen, Canada) at $1: 5000$ dilution in PBST or prostate-specific antigen (PSA, Dako, CA, USA) at 1:2000 dilution. After an hour of incubation with the indicated antibodies, the membrane was washed as above, followed by a second anti-rabbit/mouse horseradish peroxidase antibody (1:100 000) incubation for an additional hour. Protein detection was performed with SuperDura chemiluminescence reagent as per the manufacturer's instructions (Pierce, IL, USA) and visualised with a digital camera. For quantitation, the various bands were analysed with AlphaEaseFC Software version 3.1 (Alpha Innotech Corporation, San Leandro, CA, USA).

\section{Animal studies}

All studies were approved by The Mayo Foundation Institutional Animal Care and Use Committee. Male C57BL/6 mice, 5-6 weeks of age were obtained from Jackson Laboratory and housed in the Mayo Animal Resources Facilities under controlled temperature, humidity, and a $12 \mathrm{~h}$ light and dark cycle with food and water at libitum in a virus-free environment. Eight mice per group were used for each study. TRAMP-C2 cells were transfected with either pcDNA3.1 + Hsp70 or empty vector and collected $24 \mathrm{~h}$ post-transfection as described, and irradiated (10000 rads) and injected subcutaneously. Three separate injections were performed 3 days apart. Each mouse received $1 \times 10^{6}$ cells per injection. At 10 days after the last injection, mice were challenged with $3 \times 10^{6}$ wild-type TRAMP-C2 cells on the opposite flank. In the second study, stably transfected TRAMP-C2 cells were collected, irradiated (10000 rads), and injected into mice as above. At 10 days after the last injection, mice were challenged with $3 \times 10^{6}$ wild-type TRAMP-C2 cells on the opposite flank. Animals were examined and tumours were measured in three dimensions every other day using a caliper. Tumour volume was calculated, $V=$ (length)(width)(depth). Animals were removed from the study when tumour diameter was greater than $1 \mathrm{~cm}$.

\section{Statistics}

Data from the animal studies were analysed by log-rank test or Wilcoxon's signed-rank test as described in the figures. $P$-values $<0.05$ were considered to be statistically significant.

\section{RESULTS}

\section{Forced overexpression of Hsp70 can increase Hsp70 secretion}

During our studies on hsp's, we observed that Hsp70 is present in the routine culture media of TRAMP-C2 cells (data not shown). To test if the presence of Hsp70 in the spent media is a dynamic process and if overexpression can increase its secretion, we 
transiently transfected TRAMP-C2 cells with a vector coding for murine Hsp70 (pcDNA3.1 + Hsp70). As shown in Figure 1, Hsp70 is detected in both the spent media and the cytosol of transfected and mock-transfected cells. However, increased levels of Hsp70 were noted only in the spent media of transfected cells, while intracellular Hsp70 remained relatively constant over time (Figure $1 \mathrm{a}$ and $\mathrm{b}$ ). The relative amounts of Hsp70 in the supernatants and cell extracts were calculated, and greater percentages of Hsp70 were noted in the supernatants of the transfected samples $(24,48$, and $72 \mathrm{~h}$ ) when compared to the mock-transfected samples (Figure 1c).

\section{Hsp70 in supernatant is not due to cell leakage}

To eliminate the possibility of nonspecific cell leakage by physical damage, TRAMP-C2 cells from the above experiment were cotransfected with pcDNA3.1 + Hsp70 and a vector containing

a

\begin{tabular}{rrrrrrrr} 
Transfection & \multicolumn{3}{c}{ Hsp70 } & \multicolumn{3}{c}{ Mock } \\
Hours & 24 & 48 & 72 & 24 & 48 & 72 \\
\hline
\end{tabular}

b

\begin{tabular}{|c|c|c|c|c|c|c|}
\hline Transfection & & Isp7c & & & Mock & \\
\hline Hours & 24 & 48 & 72 & 24 & 48 & 72 \\
\hline
\end{tabular}

C

\begin{tabular}{ccccccc} 
Transfection & \multicolumn{3}{c}{ Hsp70 } & \multicolumn{3}{c}{ Mock } \\
\hline Hours & 24 & 48 & 72 & 24 & 48 & 72 \\
\hline Sup (\%) & 64 & 83 & 85 & 11 & 35 & 52 \\
CE (\%) & 35 & 17 & 15 & 89 & 65 & 48
\end{tabular}

d

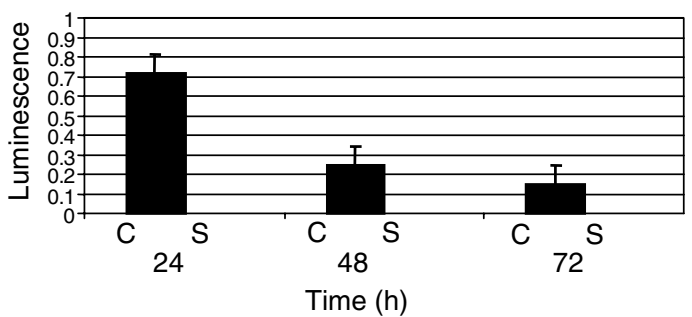

e

\begin{tabular}{cccc} 
Transfection & \multicolumn{3}{c}{ Renilla luciferase } \\
\hline Hours & 24 & 48 & 72 \\
\hline Sup (\%) & 0 & 0 & 0 \\
CE (\%) & 100 & 100 & 100
\end{tabular}

Figure I (A) Western analysis for Hsp70 in supernatants of pcDNA3.I + Hsp70 and Renilla luciferase or mock-transfected TRAMPC2 cells. Spent media were collected at 24, 48, and $72 \mathrm{~h}$ and concentrated as in Materials and methods. (B) Western analysis for Hsp70 in cell extracts of Hsp70, Renilla luciferase, and mock-transfected TRAMP-C2 cells. Ponseau S was used for normalisation. (C) Percent of Hsp70 in supernatants (Sup) and cell extracts (CE) of Hsp70 and mock-transfected TRAMP-C2 cells as determined by densitometry. (D). Comparison of luciferase activity in cell extracts (C) vs supernatants (S) in C2 cells transfected with pcDNA3.I + Hsp70 and Renilla luciferase at various time points. Spent media and whole-cell protein extracts were prepared as above. (E) Percentage of renilla protein was determined by luminescence. the cytoplasmic Renilla luciferase as a reporter. Luminescence was used to quantitate the relative amount of renilla protein in the supernatants and cell extracts. No luciferase enzyme activity was detected in the supernatants at any time points (Figure 1d). In addition, results were adjusted to account for concentrated supernatants and represented in percentages (Figure 1e).

\section{Hsp70 secretion can be found in other human prostate cell lines}

Our findings in TRAMP-C2 cells raised the question as to whether Hsp70 secretion is occurring in other prostate cell lines. We examined the spent media of various spontaneous prostate adenocarcinoma cell lines, including LAPC-4, PC-3, CWR-22, and $\mathrm{LNCaP}$ cells, and two additional transformed human prostate cell lines, RWPE-1 and RWPE-2. Note that RWPE-1 is not tumorigenic in athymic mice. RWPE-2, derived from RWPE-1, further transformed by Ki-Ras oncogene, is tumorigenic. Western analysis of these human prostate cell lines incubated under routine cell culture conditions was positive for Hsp70 in the supernatants and cell extracts, strongly suggesting that Hsp70 is secreted (Figure 2).

\section{Hsp70 secretion is not blocked by a secretion inhibitor, BFA}

To determine if the observed secreted Hsp70 was through the classical secretory pathway, BFA was used to study the secretion of Hsp70 and PSA in LNCaP cells. At $16 \mathrm{~h}$ after treatment, supernatants and cell extracts were collected as above. Western analysis for Hsp70 was performed with PSA as a positive control. LNCaP cells provided a useful model because they are an androgen-responsive human prostate adenocarcinoma cell line that expresses androgen-inducible genes such as PSA (Murtha et al, 1993). Prostate-specific antigen is a classical secretory protein that has been well studied, and its secretion and production have been shown to be inhibited by BFA (Gau et al, 1997; Konno et al, 1998). As shown in Figure 3, Hsp70 was detected in both supernatants and cell extracts and is not decreased with the addition of BFA, while a decrease in PSA secretion was noted at a concentration of $0.5 \mu \mathrm{g} \mathrm{ml}^{-1}$ BFA or higher. The presence of tubulin in the cell extracts but to only a small extent in the supernatants indicates that the Hsp70 detected in the supernatants is not due to cell death and lysis.

Forced overexpression of Hsp70 from TRAMP-C2 cells delays tumour growth and extends survival of C57BL/6 male mice

Transient overexpression experiment To test whether forced oversecretion of Hsp70 from prostate cancer cells can provide protection from tumour growth in vivo, TRAMP-C2 cells were transiently transfected with pcDNA3.1+Hsp70 and injected subcutaneously into syngeneic C57BL/6 male mice.

\section{LAPC-4 PC-3 CWR-22 RWPE-1 RWPE-2 LNCaP

$\begin{array}{lllllllllllllllllllllllll}S & C & S & C & S & C & S & C & S & C & S & C\end{array}$

Hsp70

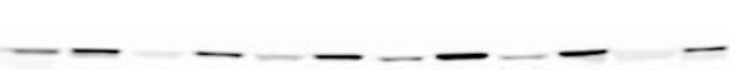

Figure 2 Western analysis for Hsp70 in supernatants (S) and cell extracts (C) of various prostate cell lines. Whole-cell protein extracts and spent media were collected at $48 \mathrm{~h}$ after being plated and subjected to Western analysis. Proteins were quantified with Bradford assay and equivalent amounts of proteins were loaded onto SDS-PAGE gel. 


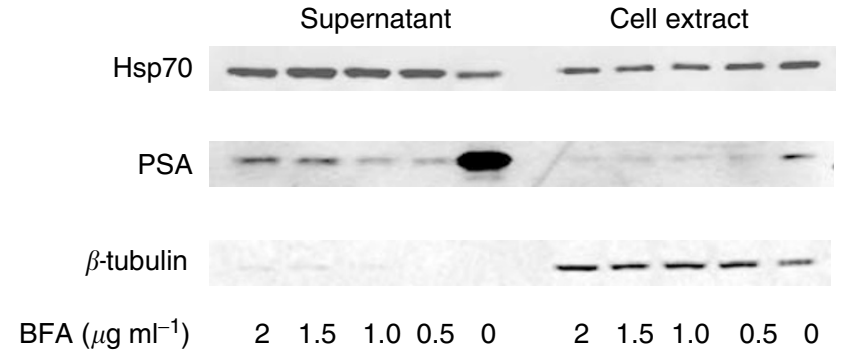

Figure 3 Western analysis for Hsp70, PSA and $\beta$-tubulin in supernatant and cell extract of LNCaP cells. Whole-cell protein extracts and spent media were prepared $16 \mathrm{~h}$ after treatment with various concentrations of BFA $\left(\mu \mathrm{g} \mathrm{ml}{ }^{-}\right)$. Protein concentrations were quantified and equal amounts of proteins were loaded onto a single gel.

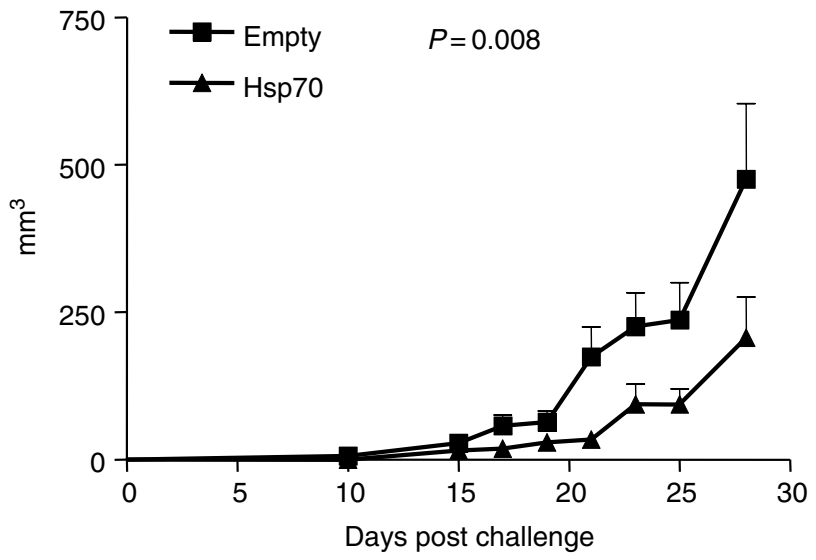

Figure 4 TRAMP-C2 tumour growth rate in C57BL/6 male mice after injecting with transiently transfected TRAMP-C2 cells with Hsp70 or empty vector. Tumours were measured every other day. $P$-values of $<0.05$ were considered statistically significant.

Cells transfected with empty vector were used as a control. At 10 days after last injection, mice were challenged with nontransfected TRAMP-C2 cells on the opposite flank. As shown in Figure 4, there is a delay in TRAMP-C2 cell growth in mice previously inoculated with Hsp70-expressing C2 cells. Statistical significance was observed at $P=0.008$ as analysed by Wilcoxon's signed-rank test between the groups (Figure 4). We also examined survival as defined by the time until the diameter of the tumour was greater than $1 \mathrm{~cm}$. We found that there was a difference in the survival rates between the two groups that was not statistically significant (Figure 5, $P=0.11$ ). This experiment suggests a protective effect offered by inoculation with cells forced to overexpress Hsp70.

\section{Stable overexpression experiment}

Furthermore, stable TRAMP-C2 transfectants with pcDNA3.1 + Hsp70 or empty vector as a control were used to reproduce the above experiment. Western analysis of these clones verified an increase in intra- and extracellular Hsp70. Injections with stable clones and subsequent live, nontransfected TRAMP-C2 challenge were performed as above. As shown in Figure 6, there was a statistically significant delay in TRAMP-C2 tumour growth in mice previously injected with Hsp70-expressing stable clones $(P=0.001)$. In addition, in this experiment there was a significant difference in survival between the two study groups (Figure 7, $P=0.02)$.

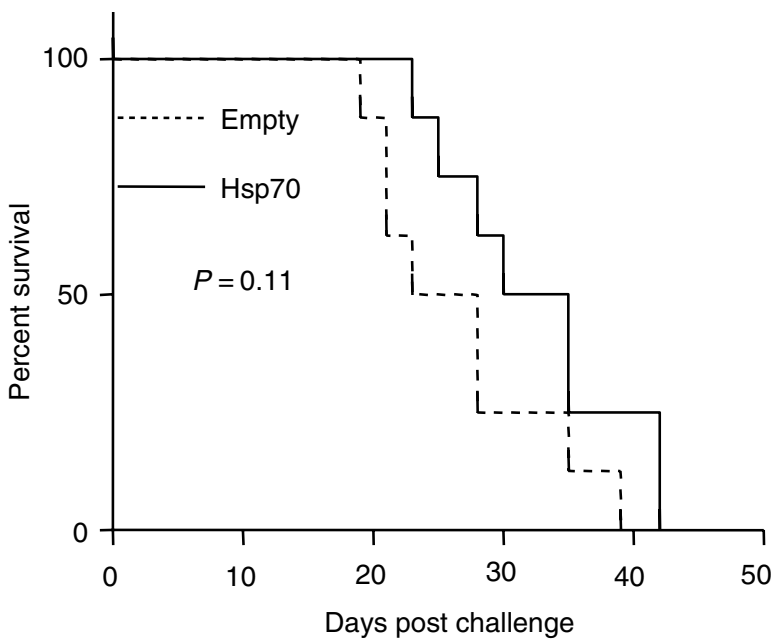

Figure 5 Percent survival of mice with transiently transfected Hsp70 TRAMP-C2 cells or empty vector.

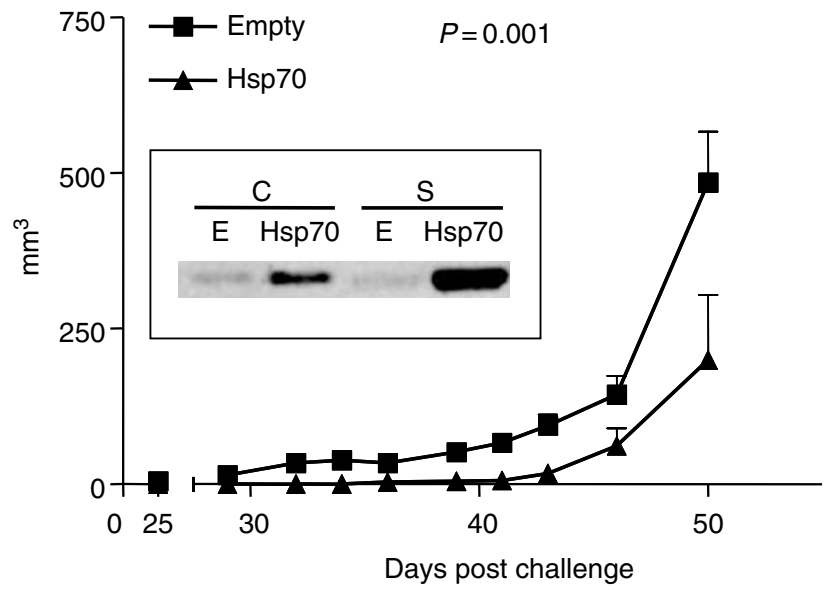

Figure 6 TRAMP-C2 tumour growth rate in C57BL/6 male mice after injecting with stably transfected Hsp70 TRAMP-C2 cells. Tumours were measured every other day. P-values of $<0.05$ were considered statistically significant. Inlet: Western analysis of Hsp70 in pcDNA3.I + murine Hsp70 (Hsp70)- and pcDNA3.I (E)-transfected stable clones. Cell extracts (C) and supernatants $(\mathrm{S})$

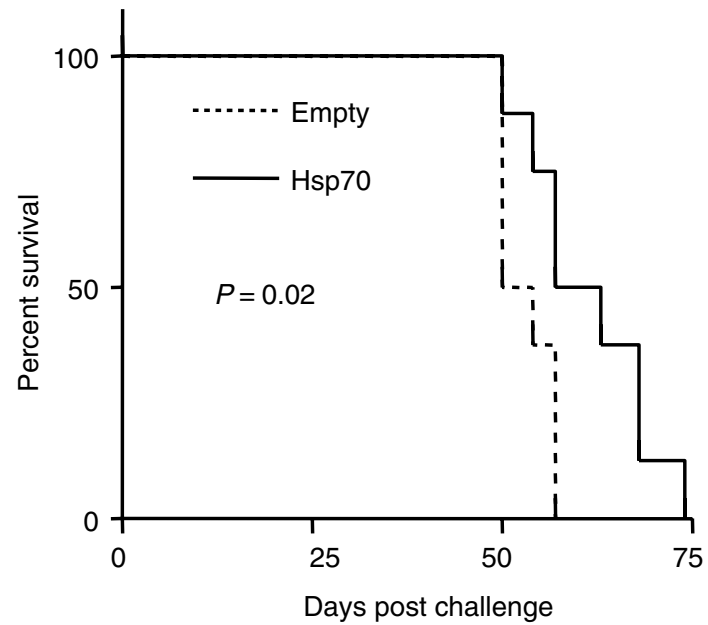

Figure 7 Percent survival of mice with stably transfected Hsp70 TRAMP-C2 cells or empty vector. 


\section{DISCUSSION}

In the course of extending our previous studies on hsp's in prostate cancer, we found that Hsp70 is secreted into the spent media by some prostate cell lines under routine cell culture conditions, although a rigorous examination of all the cell lines remains to be completed. The significance of in vivo secretion of Hsp70 remains to be further elucidated. Previous studies have shown the release of hsp's into the cultured media by rat and chick embryo cells, squid glial cells, and yeast following heat shock (Hightower and Guidon, 1989; Russo et al, 1992; Guzhova et al, 2001). It is believed that these hsp's are important in cell proliferation during embryo morphogenesis, in addition to acting as protective factors for the surrounding cells in the presence of environmental stress (Hightower and Guidon, 1989; Russo et al, 1992; Guzhova et al, 2001). Our extensive literature search indicates that this is the first time that the Hsp70 secretion is documented in mouse and human prostate cancer cells. In addition, Hsp70 secretion can be increased with overexpression. This raised an interesting implication in that this oversecretion might have the potential to be utilised in generating antitumour immunity.

Hsp studies have attempted to elucidate the means by which intracellular hsp's can interact with extracellular immune cells. One of the possibilities is that hsp's are released during tumour cell necrosis, leading to the induction of immune response. Studies by Melcher et al, utilising the suicide gene transfer system, herpes simplex virus thymidine kinase/gancyclovir (HSVtk/GCV), noted different patterns of cell death in various tumour cells. Herpes simplex virus thymidine kinase/gancyclovir utilises the strategy in which a gene coding for a prodrug-converting enzyme is delivered into tumour cells, followed by the administration of the prodrug. Thus, the enzyme converts the prodrug into a toxic compound that kills the cells (Vile et al, 1997). In this particular study, cells that became necrotic with HSVtk/GCV treatments were found to express higher levels of hsp's mRNA when compared to cells that were apoptotic. Follow-up in vivo studies showed a decrease in tumorigenicity of hsp-transfected cells (Melcher et al, 1998). Studies subjecting tumour cells to rapid freeze-thaw cyles to mimic necrosis also noted an increase in hsp's in the cell lysates and supernatants, with corresponding decrease in tumorigenicity. Studies also supported cell surface expression as an avenue by which hsp's can interact with extracellular immune cells. Studies utilising membrane-bound hsp constructs noted an increase in immunogenicity of transfected cells (Wu et al, 1999; Chen et al, 2002). Recently, a study demonstrated a decrease in tumorigenicity of hsp110-overexpressing colon cancer cells (Wang et al, 2002). Further studies should clarify the role each of these mechanisms has on immunogenicity.

\section{REFERENCES}

Bello D, Webber MM, Kleinman HK, Wartinger DD, Rhim JS (1997) Acinar differentiation by non-malignant immortalized human prostate epithelial cells and its loss by malignant cells. Carcinogenesis 18: $1225-1231$

Binder RJ, Han DK, Srivastava P (2000) CD91 is a receptor for heat shock protein gp96. Nat Immunol 1: 151-155

Botzler C, Li G, Issels RD, Multhoff G (1998) Definition of extracellular localized epitopes of Hsp70 involved in an NK immune response. Cell Stress Chaperon 3: 6-11

Cavallo F, Pierro FD, Giovarelli M, Gulino A, Vacca A, Stoppacciaro A, Forni M, Modesti A, Forni G (1993) Protective and curative potential of vaccination with interleukin-2 gene transfected cells from a spontaneous mouse mammary adenocarcinoma. Cancer Res 53: 5067-5070

Cayeux S, Richter G, Noffz G, Dorken B, Blankenstein T (1997) Influence of gene modified (IL-7, IL-4, and B7) tumor cell vaccines on tumor antigen presentation. J Immunol 158: 2834-2841
Although Hsp70 has been regarded as an intracellular protein, we found its presence in the extracellular media despite the addition of BFA, a reversible inhibitor that blocks protein translocation at the level of the endoplasmic reticulum-Golgi juncture and the trans-Golgi network (Schatz and Dobberstein, 1996; Cleves, 1997). Our findings indicate that these prostate cancer cells secrete Hsp70 via a mechanism other than the wellstudied classic vesicular secretory pathway. This 'nonclassical' secretion of proteins that lack a typical N-terminal signal peptide has been observed in several other proteins such as fibroblast growth factors 1 and 2, interleukin-1, and viral proteins (herpes simplex tegument proteins) (Cleves, 1997). The fact that Hsp70 is released into the culture media by prostate cells without known stressors, coupled with its anticancer activity raised some interesting questions: first, whether hsp's are secreted in vivo by prostate cancer cells, second and more importantly, how does this phenomena fit into the evolution of host tolerance to cancer cells.

In order to test our hypothesis that Hsp70 oversecretion from prostate cancer cells can potentially be utilised as an anticancer agent, murine Hsp70 was overexpressed in TRAMP-C2 cells and tested in vivo. TRAMP-C2 cells, a transplantable murine epithelial prostate cancer cell line, provide a useful model for the study of prostate cancer therapies (Greenberg et al, 1995). Our study showed that there is a significant decrease in the tumorigenicity of TRAMP-C2 cells in mice injected with TRAMP-C2 cells oversecreting Hsp70, in addition to a significant difference in survival between mice injected with Hsp70 oversecreting cells and control.

We can speculate from our in vitro results and previous studies that an undefined level of Hsp70 extracellularly might be involved in cancer cell protection. This is in concordance with correlation studies that suggest hsp's as unfavourable prognostic factors for progression in some types of cancer (Nylandsted et al, 2000a, b; Lebret et al, 2003). Moreover, studies in our laboratory and others have shown that increased hsp's induces antitumour activities. A study by Podack and co-workers who constructed a gp96-Ig fusion protein noted an increase in tumour immunogencity in cells transfected with this construct, suggesting that increased hsp secretion can act as a stimulatory signal for the breaking of host immune tolerance (Yamazaki et al, 1999). Additional studies of hsp secretion will likely offer insights and help us to answer fundamental immunologic questions with respect to the development of tolerance and immunity.

\section{ACKNOWLEDGEMENTS}

This work was funded by Department of Defense grants DAMD1701-1-0074 and DAMD17-98-18523.
Chen X, Tao QT, Yu H, Zhang L, Cao X (2002) Tumor cell membrane bound heat shock protein 70 elicits antitumor immunity. Immunol Lett 84: $81-87$

Cleves AE (1997) Protein transports: the nonclassical ins and outs. Curr Biol 7: R318-20

Fearon ER, Pardoll DM, Itaya T, Golumbek P, Levitsky HI, Simons JW, Karasuyama H, Vogelstein B, Frost P (1990) Interleukin-2 production by tumor cells bypasses $\mathrm{T}$ helper function in the generation of an antitumor response. Cell 60: 397-403

Foster BA, Gingrich JR, Kwon ED, Madias C, Greenberg NM (1997) Characterization of prostatic epithelial cell lines derived from transgenic adenocarcinoma of the mouse prostate (TRAMP) model. Cancer Res 57: $3325-3330$

Gau JT, Salter RD, Krill D, Gove ML, Becich MJ (1997) The biosynthesis and secretion of prostate-specific antigen in LNCaP cells. Cancer Res 57: $3830-3834$ 
Gething MJ, Sambrook J (1992) Protein folding in the cell. Nature 355: $33-45$

Greenberg N, DeMayo F, Finegold M, Tilley W, Aspinall J (1995) Prostate cancer in a transgenic mouse model. Proc Natl Acad Sci USA 92: 3439

Guzhova I, Kislyakova K, Moskaliova O, Fridlanskaya I, Tytell M, Cheetham $\mathrm{M}$, Margulis B (2001) In vitro studies show that Hsp70 can be released by glia and that exogenous Hsp70 can enhance neuronal stress tolerance. Brain Res 914: 66-73

Hightower LE, Guidon Jr PT (1989) Selective release from cultured mammalian cells of heat-shock (stress) proteins that resemble glia-axon transfer proteins. J Cell Physiol 138: 257-266

Houghton AN (1994) Cancer antigens: immune recognition of self and altered self. [comment]. J Exp Med 180: 1-4

Ishii $\mathrm{T}$, Udono $\mathrm{H}$, Yamano $\mathrm{T}$, Ohta $\mathrm{H}$, Uenaka $\mathrm{A}$, Ono $\mathrm{T}$, Hizuta $\mathrm{A}$, Tanaka N, Srivastava PK, Nakayama E (1999) Isolation of MHC class I-restricted tumor antigen peptide and its precursors associated with heat shock proteins hsp70, hsp90, and gp96. J Immunol 162: $1303-1309$

Konno S, Mordente JA, Chen YP, Wu JM, Tazaki H, Mallouh C (1998) Effects of brefeldin A on androgen receptor-mediated cellular responses in human prostatic carcinoma LNCaP cells. Mol Urol 2: 7-11

Lebret T, Watson RW, Fitzpatrick JM (2003) Heat shock proteins: their role in urological tumors. J Urol 169: $338-346$

Mahler C, Denis L (1992) Management of relapsing disease in prostate cancer. Cancer 70: 334-329

Matsutake T, Srivastava P (1999) CC91 is involved in MHC class II presentation of gp96 chaperoned peptides. Cell Stress Chaperon 5: 378

Melcher A, Todryk S, Hardwick N, Ford M, Jacobson M, Vile RG (1998) Tumor immunogenicity is determined by the mechanism of cell death via induction of heat shock protein expression. Nat Med 4: $581-587$

Murtha P, Tindall DJ, Young C (1993) Androgen induction of a human prostate specific kallikrein, hKLK2: characterization of an androgen response element in the $5^{\prime}$ region of the gene. Biochemistry 32: $6459-6464$

Nylandsted J, Brand K, Jaattela M (2000a) Heat shock protein 70 is required for the survival of cancer cells. Ann NY Acad Sci 926: $122-125$

Nylandsted J, Rohde M, Brand K, Bastholm L, Elling F, Jaattela M (2000b) Selective depletion of heat shock protein 70 (Hsp70) activates a tumorspecific death program that is independent of caspases and bypasses Bcl-2. Proc Natl Acad Sci USA 97: 7871-7876

Panjwani N, Popova L, Febbraio M, Srivastava P (2000) The CD36 scavenger receptor as a receptor for gp96. Cell Stress Chaperon 5: 391
Parsell DA, Lindquist S (1993) The function of heat-shock proteins in stress tolerance: degradation and reactivation of damaged proteins. Annu Rev Genet 27: 437-496

Ponomarev ED, Tarasenko TN, Sapozhnikov AM (2000) Splenic cytotoxic cells recognize surface HSP70 on culture-adapted EL-4 mouse lymphoma cells. Immunol Lett 74: 133 - 139

Russo P, Kalkkinen N, Sareneva H, Paakkola J, Makarow M (1992) A heat shock gene from Saccharomyces cerevisiae encoding a secretory glycoprotein. Proc Natl Acad Sci USA 89: 8857

Sanda MG, Ayyagari SR, Jaffee EM, Epstein JI, Clift SL, Cohen LK, Dranoff G, Pardoll DM, Mulligan RC, Simons JW (1994) Demonstration of a rational strategy for human prostate cancer gene therapy. J Urol 151: 622-628

Schatz G, Dobberstein B (1996) Common principles of protein translocation across membranes. Science 271: 1519-1526

Srivastava PK, Menoret A, Basu S, Binder RJ, McQuade KL (1998) Heat shock proteins come of age: primitive functions acquire new roles in an adaptive world. Immunity 8: 657-665

Suto R, Srivastava P (1995) A mechanism for the specific immunogenicity of heat shock protein-chaperoned peptides. Science 269: 1585-1588

Vanaja DK, Grossmann ME, Celis E, Young CY (2000) Tumor prevention and antitumor immunity with heat shock protein 70 induced by 15-deoxy-delta12,14-prostaglandin J2 in transgenic adenocarcinoma of mouse prostate cells. Cancer Res 60: 4714-4718

Vieweg J, Rosenthal FM, Bannerji R, Heston WD, Fair WR, Gansbacher B, Gilboa E (1994) Immunotherapy of prostate cancer in the Dunning rat model: use of cytokine gene modified tumor vaccines. Cancer Res 54: $1760-1765$

Vile RG, Castleden S, Marshall J, Camplejohn R, Upton C, Chong H (1997) Generation of an anti-tumor immune response in a non-immunogenic tumor:HSVtk killing in vivo stimulates a mononuclear cell infiltrate and a Th1-like profile of intratumoral cytokine expression. Intl J Cancer 71: $267-274$

Wang XY, Li Y, Manjili MH, Repasky EA, Pardoll DM, Subjeck JR (2002) Hsp110 over-expression increases the immunogenicity of the murine CT26 colon tumor. Cancer Immunol Immunother 51: 311-319

Wang Y, Kelly CG, Karttunen JT, Whittall T, Lehner PJ, Duncan L, MacAry P, Yonnson JS, Singh M, Oelmann W, Cheng G, Bergmeier L, Lehner T (2001) CD40 is a cellular receptor mediating mycobaterial heat shock protein 70 stimulation of CC chemokines. Immunity 15: 971-983

Wu WZ, Liu KD, Xie Q, Wu HS (1999) The study on the expression of membrane HSP70 protein in $\mathrm{H} 22$ cell and its immunoprotective mechanism against carcinoma. J Exp Clin Cancer Res 18: 543-548

Yamazaki K, Nguyen T, Podack ER (1999) Cutting edge: tumor secreted heat shock-fusion protein elicits CD8 cells for rejection. J Immunol 163: $5178-5182$ 